\title{
HEMODIALYSIS PATIENT'S AWARENESS ABOUT KIDNEY TRANSPLANTATION
}

\author{
Veselina Vasileva, Mariana Dimitrova \\ Department of Nursing Copare, Faculty of Public Health, Medical University of Varna
}

\begin{abstract}
Kidney transplantation is the most frequent type of transplantation worldwide and within the country. It is the only effective treatment for patients diagnosed with chronic renal failure. Currently there are approximately $\mathbf{3 0 0 0}$ patients in Bulgaria on hemodialysis. According to the latest data of the Executive Agency for Transplantation (EAT), 977 persons are waiting for a new kidney. The main reason for the low number of enlisted patients for renal transplantation is mostly due to the lack of information about their options.

AIM: The purpose of this study is to explore the views of the 50 patients on hemodialysis, their awareness of transplantation possibilities as a factor for improving the quality of life and increase the level of interest about the procedure.

MATERIALS AND METHODS: A total of 50 patients undergoing hemodialysis treatment at the St. Marina University Hospital in Varna have been interviewed using a questionnaire method and an individual anonymous survey. The survey was conducted in July 2016.

RESULTS AND CONCLUSIONS: The importance of the problem was confirmed by the fact that almost half of the respondents assessed their awareness about transplantations as low. After recognizing the need to raise their awareness of the problem, significantly higher percentage $(83 \%)$ of the patients stated their motivation to learn about new developments in the field of transplantation through specialized trainings on the subject.

The survey data reflects the views of the respondents who increased their knowledge on organ transplantation and realized the possibilities of improving the quality of their life.
\end{abstract}

Keywords: awareness, kidney transplantation, patient, hemodialysis, quality of life

Address for correspondence:

Veselina Vasileva

Faculty of Public Health

Medical University of Varna

55 Marin Drinov St

9002 Varna

e-mail: veselina.vasileva@mu-varna.bg

\section{INTRODUCTION}

Transplantation is a surgical intervention that takes healthy organs or tissues from one body (the donor) and grafts them onto another body (recipient). Grafts (transplants) have the full function of the problematic organ, thus prolonging and improving the quality of life of transplant recipients. The transplant can be both from an alive or deceased donor. The main legal document, which regulates the terms and conditions for carrying out the transplantation of organs, tissues and cells in human medicine in the Republic of Bulgaria has been in effect since 
01.01.2004, last modified - amended and supplemented by SG, issue 15 from 15 February $2013(1,2)$.

Renal transplantation is a leader among the various types of transplants, both globally and nationally $(3,4)$. The procedure is an effective method for the treatment of patients diagnosed with chronic renal failure in a terminal stage. With such a diagnosis in Bulgaria are about 3,000 patients including those undergoing a hemodialysis treatment. The importance of the problem is confirmed by the fact that according to the Executive Agency for Transplantation (EAT), the number of potential recipients of a kidney to date (11.07.2016) is 977 (4). It is interesting to note that approximately $2 \%$ of the patients on hemodialysis are at the St. Marina University Hospital in Varna, which is the best center serving patients on hemodialysis treatment in the country. The relatively low proportion of patients included in the kidney waiting list is a complex issue, at the heart of which stand issues related to treatment options. The low awareness of the problem puts patients in social seclusion. In this context, achieving and maintaining optimal quality of life proved to be a difficult target, the psychological and social aspects of which are insufficiently supported.

\section{AIM}

The purpose of this study was to explore the views of the 50 patients undergoing hemodialysis, the importance of awareness of transplantation as a factor for improving the quality of life in patients awaiting kidney transplantation and increasing the interest in the procedure.

\section{TASKS}

1. To explore the views of the patients undergoing hemodialysis on the need to raise awareness regarding opportunities for effective treatment.

2. To establish the level of awareness of respondents about the issue through personal self-evaluation.

3. To examine the accuracy and reliability of the information on the topic by analyzing the literature used by patients; to analyze the accuracy and reliability of the literature on the topic used for informing the patients.
4. To explore the attitudes of patients on options for raising the awareness of patients about transplants.

\section{MATERIALS AND METHODS}

We have used a questionnaire method and an individual anonymous survey. We have examined the opinion of 50 patients undergoing hemodialysis treatment at the Hemodialysis Clinic at the St. Marina University Hospital in Varna. The age of the respondents was between 29 and 73. The survey was conducted during the month of July 2016

The analysis of the primary sociological information used Microsoft Excel 2013. This scientific research has a representative character.

\section{RESULTS}

Patients diagnosed with chronic renal failure require the maintenance of a high quality of life, which ensures good health and extends life expectancy. This is a complex process at the base of which stands high awareness of the disease and treatment options. The importance of the problem was confirmed by the fact that almost half of the respondents assessed their awareness about transplants as low (45.80\%), (Fig. 1).

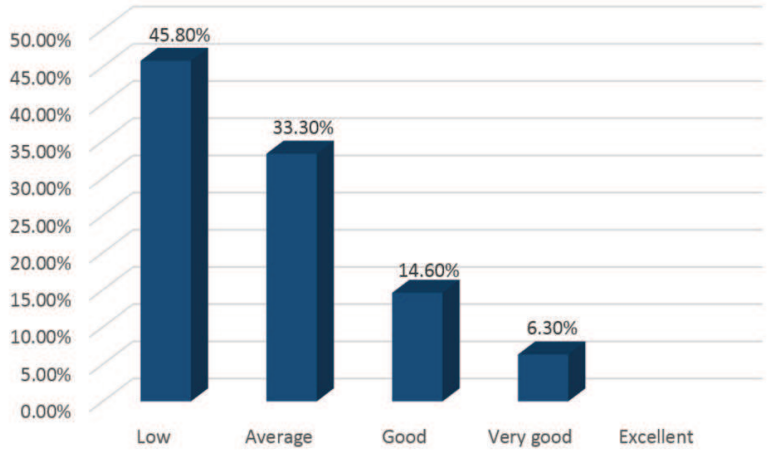

Fig. 1. Awareness about transplants

Patients defining their knowledge on the topic as average were $33.30 \%$ of the total number surveyed. A relatively low share, considered that they have good basic knowledge of the issues related to treatment options (14.60\%). Approximately $6 \%$ of respondents define their awareness of the issue as very good. There 
are no data for patients designating themselves as excellently informed on transplants.

Once low self-esteem is established among respondents a detailed study of the problem should be conducted by analyzing the sources, which use patient awareness on the topic. The survey data show that the most valuable source of information for the surveyed groups is the medical team (51.00\%), (Fig. 2).

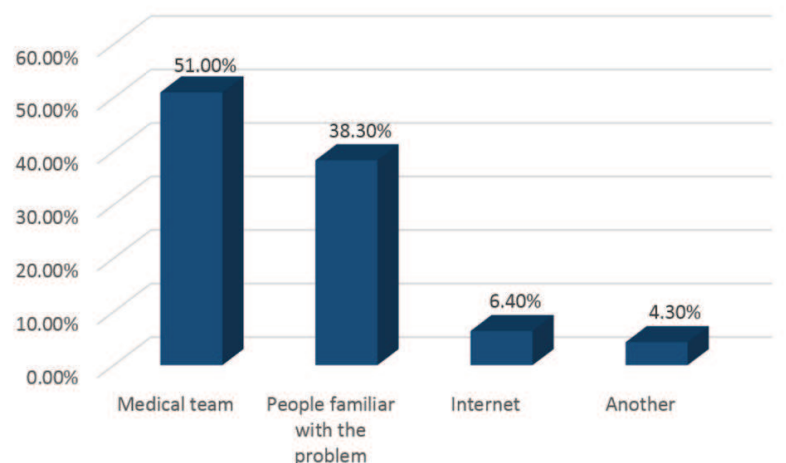

Fig. 2. Distribution of sources of information on the subject, according to the value of their information for patients

This relatively high percentage provokes an indepth analysis on the role of the individual medical professionals in this process. The survey results indicated that the most valuable source of information from the medical team was the nurse with $56.30 \%$, followed by the doctor with $28.10 \%$, and in third position, with 15.60 percent, the respondents put other sources than those specified. There was no information on the role of the transplantation coordinator in the process of providing awareness on issues related to transplants.

Approximately $40 \%$ of respondents indicated that the most valuable information for them was obtained from someone familiar with this problem (38.30\%). The next most popular response scored 6.40 percent of the patients citing the Internet as a valuable information source. Only $4 \%$ of respondents identified other sources than those listed but none of them has indicated specifically from whom or from where they obtained this information, which in turn again generated interest about the nature of the source.
The education of patients and their families about the disease and treatment options is part of the duties of medical personnel. The form in which it was organized was usually a health-related talk, which was confirmed by the majority of the respondents (70.40\%). The remaining respondents claimed that their training had other form than those listed here, but there were no answers on what it was specifically (29.60\%). According to data from this research, there are no reports of patient involvement in special-

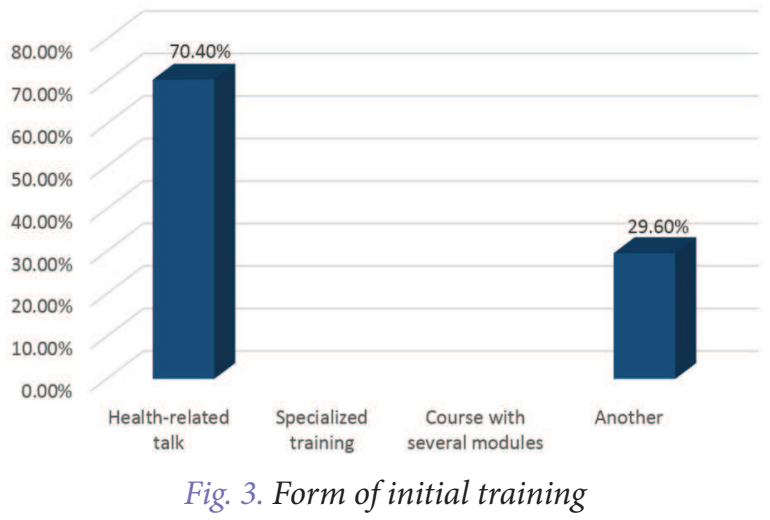

ized training and modular courses, which in turn necessitates an analysis of the reasons (Fig. 3).

Transplantation medicine is a dynamic science, which is why it was examined together with the attidute of patients to developments in the field. The survey results expressed lack of interest from $27.70 \%$ of the respondents and $25.50 \%$ were relatively uninformed on the issue. The remaining respondents exhibited some interest, as $17 \%$ followed some developments in the field of transplantation, and $29.80 \%$

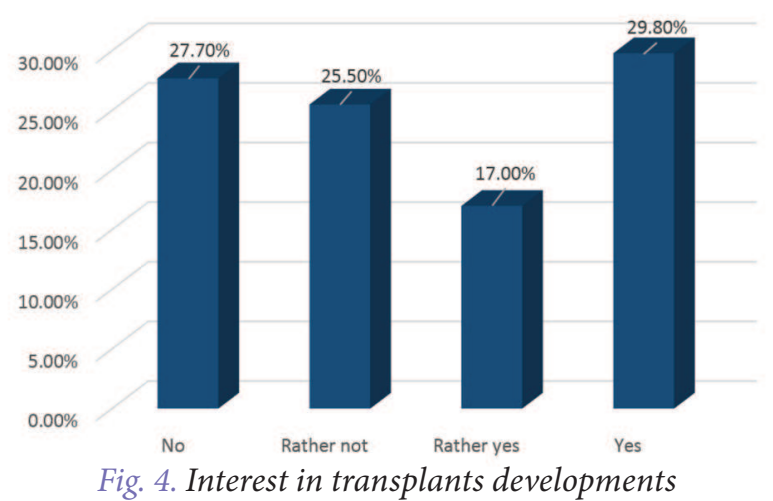


were actively seeking an adequate solution to their problem (Fig. 4).

The majority included patients on the waiting list for kidney transplants. Their level of awareness was maintained through literature of a different nature. The preferred form confirmed by the research was the Internet (26.10\%), followed by other than those specified (47.80\%) and lastly the medical team (26.10\%). No data are available for obtaining information through seminars on the topic, which in turn supports the fact that patients do not attend such forms of training.

The awareness of hemodialysis patients about treatment options should be continuously updated. A significantly higher percentage recognized the need to raise their awareness about the problem and considered that the holding of specialized training would be beneficial both for themselves and for their loved ones (83.30\%). The remaining $16.70 \%$ of the respondents did not have an opinion on the presented

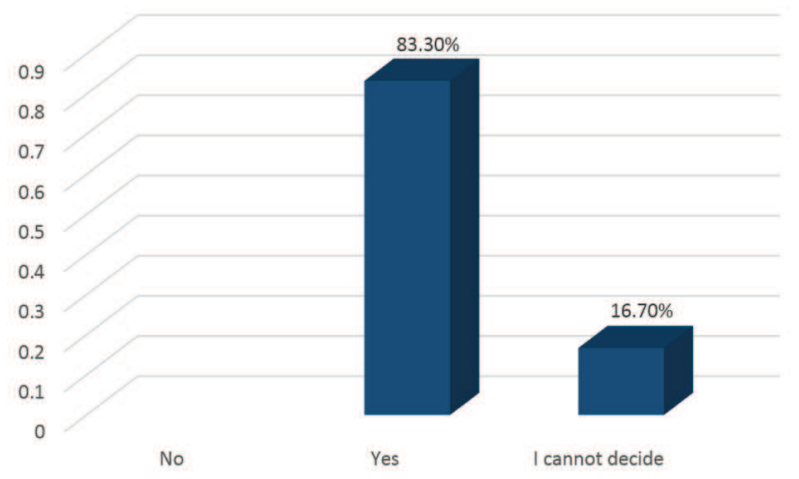

Fig. 5. Support for conducting specialized training for patients and their families about organ transplants

idea. There are no data for disapproval of any initiatives focused on informing the patients (Fig. 5).

\section{DISCUSSION}

Low awareness of patients about treatment options is a prerequisite for lowering their quality of life, which subsequently leads to the emergence of a number of complications and poor health. The problem requires high commitment from the medical team and given that one third of the study group patients were included in the waiting list for kidney transplant and maintaining optimal health is particularly important.
The low level of awareness on the subject among patients is a problem with a complex character at the heart of which stands the lack of information (45.80\%). This provokes in - depth analysis of references. A detailed study of sources on the subject, in terms of value of the information obtained was conducted. Healthcare professionals are the source of information about the disease and treatment options among patients and have the leading result (51.00\%). The majority of respondents define the information received from the nurse the most valuable for the patient and confirm that it gave them high confidence. Noteworthy is the second most popular answer among respondents supported by approximately $40 \%$, which determines the most valuable information to be that obtained from people familiar with this problem (38.30\%). These patients described it as useful advice from the medical experts. Now is the time to pay attention to the potential danger of disinformation due to misinterpretations and errors in the received information. When there is no clear account of the information received, an in-depth study of the level of awareness on the subject among these patients should be conducted.

The education of patients and their families about the disease and treatment options is part of the duties of the medical personnel. The form in which it is usually held is a health-related talk, a fact confirmed by the majority of respondents $(70.40 \%)$. Data from this research showed that there were no reports of patient involved in specialized training and modular courses, which in turn makes it necessary to study the specific reasons. The information from these forms would be extremely useful to patients and their relatives in the selection of appropriate treatment and maintaining optimal health in this difficult period.

The attitudes and interests of patients to developments in transplant medicine were examined. The survey results expressed lack of or low level of interest indicated by a total of $53.20 \%$ of the respondents. Lack of interest was explained by the fact that most of them were not included in the waiting list for kidney transplants and were strongly discouraged by the outcome of their disease. The remaining $46.80 \%$ monitored developments in the field of transplantation in search of an adequate solution for treatment. References for information on the topic were studied as well. The received results raised concern because of 
Veselina Vasileva, Mariana Dimitrova

lower allotted place of medical specialists among the sources that benefit patients. Since the nature of the information obtained was not clear an in-depth analysis of the level of awareness on the subject among these patients should be conducted. Data from studies on specific issues highlight the need to raise the attention and awareness of patients on the topic in order to provide the most adequate for their status options for dealing with the problem. This in turn requires a study of attitudes towards participation in various forms of education among respondents.

The idea of conducting specialized training for patients and their families about organ transplants, met high support and positive attitude on the part of respondents $(83.30 \%)$. This in turn confirms the need and desire of patients to raise awareness on the topic. From this it can be concluded that respondents recognize the importance of high level of awareness as a factor for improving the quality of life.

\section{CONCLUSION}

Most patients realize the importance of higher level awareness as a factor for improving the quality of life. As a result of self-assessment, the majority of patients saw themselves as having a low level of awareness on the issue. There is a need to check the level of awareness among patients in order to detect and correct errors. A specialized training among patients and their families is needed to improve the quality and familiarity with developments in transplant medicine.

Increased awareness of patients about the disease and treatment options is basically a process of maintaining optimal quality of life. Patients should have excellent awareness and continuously work towards promotion and personal development. Achieving the desired high performance requires active participation by medical professionals.

\section{REFERENCES}

1. Law transplant organs, tissues and cells.

OG/83/2003. all amend. and add. incl. amend. OG/41/2009. (in Bulgarian)

2. Georgieva S, Savova A, Paskalev E, Petrova D. Literary study of legislative requirements and drug therapy of renal transplant. General Medicine, Central Medical Library, MU Sofia. 2012; 14 (3): 3-11. (in Bulgarian)
3. Garcia GG, Harden P, Chapman J. The Global Role of Kidney Transplantation Kidney. Blood Press Res. 2012; 35:299-304.

4. The Republic of Bulgaria Executive Agency for Transplantation Ministry of Health. [cited 2016 July 11]. Records and statistics. Statistical data. [about 2 screens] Available from: http://bgtransplant.bg/iat/registers\%20and\%20statistics.php 